\title{
On Neutron Branching Processes
}

\author{
By \\ Takakazu Mori*, Shinzo WAtAnABE** and \\ Toshio YAMADA ${ }^{\dagger}$
}

\section{Introduction}

A statistical theory of the neutron transport process has been discussed by Harris [1] and Mullikin [7]. They considered the neutron population by generation rather than in real time and formulate them as a discretetime branching process. In this paper, we will formulate the neutron transport process as a continuous time branching process and study asymptotic properties of the neutron population. As a fundamental equation, we have a non-linear equation like (1.3) and our results may be regarded, as a problem in analysis, to be concerned with the asymptotic properties of solutions of such a non-linear equation. In such study, a linearized Boltzman equation, which is the dual of the evolution equation for expectation semigroup, plays important role. In this paper, we are mainly concerned with a monoenergetic and isotropic transport process on bounded domain in $\mathbf{R}^{3}$, though a more general case can be treated by the same method. Also, our method can be applied to a general class of branching processes including branching diffusion processes but we will not go further into such generalizations.

\section{$\S 1$. Formulation of the Neutron Branching Process and the Basic Equations}

To apply the theory of branching processes, we will give, as a model

Received December 16, 1970.

Communicated by S. Matsuura.

* Department of Mathematics, Kyoto Sangyo University, Kamigamo, Kyoto, Japan.

** Department of Mathematics, Kyoto University, Kitashirakawa, Kyoto, Japan.

† Department of Applied Sciences, Kyushu University, Hakozaki, Fukuoka, Japan. 
of neutron transport phenomenon, the following simple one. A neutron, moving straightly in a bounded homogeneous medium $D$ with unit speed, will collide with a nucleus at random time $T$ which is exponentially distributed with mean $\sigma^{-1}$. If a collision occurs, then $n$ neutrons are produced with probability $p_{n}, n=0,1,2, \ldots$ Each of new particles, the direction of which is isotropically distributed, performs a similar motion as the original one independently each other. On the other hand, a neutron is absorbed (extinct) if it leaves the medium.

Let $D$ be a bounded closed convex domain in the three dimensional Euclidean space $\mathbf{R}^{3}$ with a smooth boundary, and $\Omega$ be the unit sphere in $\mathbf{R}^{3}$. Denoting by $G$ the product space $D \times \Omega$, a neutron can be represented by a point $(x, \omega)$ in $G, x$ and $\omega$ being the position and the direction of a particle, respectively. $\partial G$ stands for the set of all points $(x, \omega)$ in $G$ such that $x$ belongs to the boundary of $D$ and $\omega$ is a direction exiting $D$; i.e. $\left(n_{x}, \omega\right) \geq 0$, where $n_{x}$ is the direction of outernormal at $x$.

Let $S^{n}$ be the $n$-fold symmetric product space of $S=G$ and $\hat{\mathbf{S}}=\bigcup_{n=0}^{\infty} S^{n}$ $\cup\{\Delta\}$ be the one point compactification of the topological sum $\bigcup_{n=0}^{\infty} S^{n}$ where $S^{0}=\{\partial\}$, ( $\partial$ is an extra point). In order to formulate the above branching process as a Markov process on $\hat{\mathbf{S}}$, it is sufficient to specify the nonbranching part $X_{t}^{0}$, which is a Markov process on $G$, and the branching system $\left\{p_{n}(z), \pi_{n}(z, d \mathbf{y}), z \in G, \mathbf{y} \in S^{n}\right\}_{n=0}^{\infty}[2]$. Intuitively, $X_{t}^{0}$ is the motion of a neutron before collision or before it leaves the domain, and branching system represents the law of new born neutrons when collision occurs; $p_{n}(z)$ is the probability that the original particle is replaced by $n$ new particles when collision occurs at $z$ and $\pi_{n}(z, d y)$ is the distribution of the position of these $n$ particles. They are given as follows; let $x_{t}$ be a uniform motion process on $\mathbf{R}^{3} \times \Omega$, i.e., a particle starting at $(x, \omega)$, is at $(x+t \omega, \omega)$ in time $t$, and $\tau^{\prime}$ be the hitting time of the process $x_{t}$ for $\partial G$.

Define $X_{t}^{0}$ as

$$
X_{t}^{0}= \begin{cases}x_{t} & ; t<\tau^{\prime} \wedge T \equiv \tau \\ \delta & ; t \geq \tau\end{cases}
$$


where $T$ is an exponential time with mean $\sigma^{-1}$ independent of the process $x_{t}$. Let, for a given probability sequence $\left\{p_{n}\right\}$,

and

$$
p_{n}(z)= \begin{cases}p_{n} & \text { if } z=(x, \omega) \in G-\partial G \\ 0 & \text { if } z \in \partial G \text { and } n \neq 0 \\ 1 & \text { if } z \in \partial G \text { and } n=0\end{cases}
$$

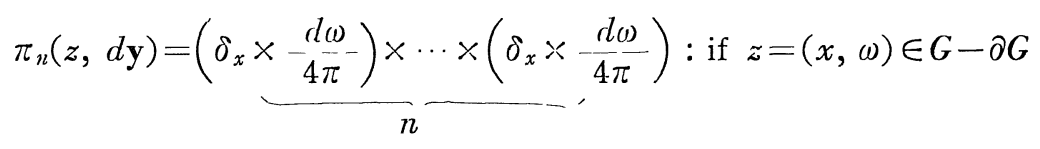

where $\delta_{x}$ is the unit measure at $x$ and $d \omega$ is the spherical Lebesgue measure on $\Omega$. By $[2]$, we can construct a branching process $\left\{X_{t}, P_{(x, \omega)}\right\}$ corresponding to $\left(X_{t}^{0}, p_{n}, \pi_{n}\right)$ on a suitable probability space, where $P_{(x, \omega)}$ is the probability law of the process under the condition that there is initially one particle at $(x, \omega) \in G$. We will adopt this branching process as our model of the neutron transport.

For every function $f(z)$ on $G, \hat{f}$ and $\breve{f}$ are functions on $\hat{\mathbf{S}}$ defined in the following way;

$$
\begin{aligned}
& \hat{f}(\boldsymbol{z})= \begin{cases}1, & \text { if } \boldsymbol{z}=\partial \text { or } \boldsymbol{z}=z \in \partial G \\
f(z) & \text { if } \boldsymbol{z}=z \in G-\partial G \\
\prod_{i=1}^{n} \hat{f}\left(z_{i}\right) & \text { if } z=\left(z_{1}, \ldots, z_{n}\right) \in S^{n}, n \geqq 2, \\
0 & \text { if } \boldsymbol{z}=\Delta\end{cases} \\
& \check{f}(\boldsymbol{z})= \begin{cases}0 & \text { if } \boldsymbol{z}=\partial \text { or } z=\boldsymbol{z} \in \partial G \\
f(z) & \text { if } \boldsymbol{z}=z \in G-\partial G \\
\sum_{i=1}^{n} \check{f}\left(z_{i}\right) & \text { if } \boldsymbol{z}=\left(z_{1}, \ldots, z_{n}\right) \in S, n \geqq 2 \\
0 & \text { if } \boldsymbol{z}=\Delta .\end{cases}
\end{aligned}
$$

Further, the following notations are introduced;

$\mathrm{C}(G),\left(\mathbf{C}_{1}(G), \mathrm{C}_{1}^{+}(G)\right)=$ the set of all continuous functions on $G$ (with sup-norm $\|f\|<1$, resp. with $\| f i<1$ and $f \geq 0)$

$\mathbf{B}(G),\left(\mathbf{B}_{1}(G), \mathbf{B}_{1}^{+}(G)\right)=$ the set of all bounded Borel measurable func- 
tions $f$ on $G$ (with $\|f\|<1$ resp. with $\|f\|<1$ and $f \geq 0$ ),

$\mathfrak{S}(G)=$ the set of all spuare integrable functions on $G$ with $L_{2}$-norm il $\|_{2}$,

$\mathrm{C}(D)=$ the set of all continuous functions on $D$,

$S_{b}(x, \omega)=\inf \{t \mid x+t \omega \notin D\}$,

$F[z]=\sum_{n=0}^{\infty} p_{n} z^{n}$

$e_{\partial}=\inf \left\{t ; X_{t}=\partial\right\} \quad$ (the extinction time)

$e_{\Delta}=\inf \left\{t ; X_{t}=\Delta\right\} \quad$ (the explosion time)

$Z_{t}^{E}=\check{I}_{E}\left\langle\left(X_{t}\right), E \in \mathbf{B}(G) \quad\left(\check{I}_{E}\right.\right.$ is the indicator function of $E$; the number of particles in $E$ at time $t$.)

$Z_{t}=Z_{t}^{G}$.

$S_{b}(x, \omega)$ is a bounded continuous function on $G$ by the assumption on the domain $D$. We shall always assume that,

( I ) $p_{0}+p_{1}<1$

(II) $F^{\prime}[1]=\sum_{n=0}^{\infty} n p_{n}<+\infty$.

From [2], the following relations hold. For every $f \in \mathbf{B}_{1}(G)$, we define

$$
u(t, x, \omega)=T_{t} \hat{f}(x, \omega)=E_{(x, \omega)}\left[\hat{f}\left(X_{t}\right)\right], \quad(x, \omega) \in G .
$$

Then $u$ satisfies

$$
u(t, x, \omega)=\left\{\begin{aligned}
e^{-\sigma t} f(x+t \omega, \omega)+\sigma \int_{0}^{t} e^{-\sigma r} F[\tilde{u}(t-r, x+r \omega)] d r, & \\
& \text { if } t<S_{b}(x, \omega), \\
e^{-\sigma S_{b}(x, \omega)}+\sigma \int_{0}^{S_{b}} e^{-\sigma r} F[\tilde{u}(t-r, x+r \omega)] d r, & \text { if } t \geq S_{b}(x, \omega),
\end{aligned}\right.
$$

where, $\tilde{u}(t, x)=\frac{1}{4 \pi} \int_{\Omega} u(t, x, \omega) d \omega$.

Further, if $f \in \mathbf{C}_{1}(G)$, then $u$ satisfies

$$
\begin{aligned}
& \left\{\begin{aligned}
\frac{\partial u(t, x, \omega)}{\partial t} & =\omega \cdot \nabla u(t, x, \omega)-\sigma u(t, x, \omega)+\sigma F[\tilde{u}(t, x)] \\
& \equiv A u(t, x, \omega)
\end{aligned}\right. \\
& \begin{array}{l}
u(t, x, \omega)=1 \quad \text { if }(x, \omega) \in \partial G \\
u(0+, x, \omega)=f(x, \omega) .
\end{array}
\end{aligned}
$$

On the other hand, if $f \in \mathbf{B}(G)$, then 


$$
v(t, x, \omega)=T_{t} \check{f}(x, \omega)=E_{(x, \omega)}\left[\check{f}\left(X_{t}\right)\right]
$$

satisfies

$$
v(t, x, \omega)=\left\{\begin{array}{r}
e^{-\sigma t} f(x+t \omega, \omega)+\sigma F^{\prime}[1] \int_{0}^{t} e^{-\sigma r} \tilde{v}(t-r x+r \omega) d r, \\
\text { if } t<S_{b}(x, \omega) \\
\sigma F^{\prime}[1] \int_{0}^{S_{b}(x, \omega)} e^{-\sigma r} \check{v}(t-r, x+r \omega) d r \\
\text { if } t \geq S_{b}(x, \omega)
\end{array}\right.
$$

where, $\tilde{v}(t, x)=\frac{1}{4 \pi} \int_{\Omega} v(t, x, \omega) d \omega$.

Further, if $f \in \mathbf{C}(G)$, then

$$
\left\{\begin{array}{l}
\frac{\partial v(t, x, \omega)}{\partial t}=\omega \cdot \Delta v(t, x, \omega)-\sigma v(t, x, \omega)+\sigma F^{\prime}[1] \tilde{v}(t, x) \equiv B v(t, x, \omega) \\
v(t, x, \omega)=0 \quad \text { if }(x, \omega) \in \partial G \\
v(0+, x, \omega)=f(x, \omega) .
\end{array}\right.
$$

Assumption (II) guarantees that the explosion does not occur in finite time, i.e. $P_{(x, \omega)}\left[e_{\Delta}=+\infty\right]=1,[2]$. If $f=X_{E}$, then $\check{f}\left(X_{t}\right)$ is the number of neutrons in $E$ at time $t$, so equation (1.5) or (1.6), which is the dual of the so-called linearlized Boltzmann transport equation, determines completely the expected number of neutrons.

\section{$\S 2$. The Extinction Probability and the Asymptotic Behaviour or Expected Number}

If we define $q(t, x, \omega)=T_{t} \hat{O}(x, \omega)$, then it is the extinction probability $P_{(x, \omega)}\left[e_{\partial} \leq t\right]$ at time $t$, starting initially at $(x, \omega)$. Obviously $q(t, x, \omega)$ is increasing in $t$, continuous in $(x, \omega)$ and $0 \leq q(t, x, \omega) \leq 1$. Hence, there exists a limit $q(x, \omega)$ as $t \rightarrow \infty$. Letting $t \rightarrow \infty$ in $(1.2), q(x, \omega)$ is a solution of

$$
u(x, \omega)=e^{-\sigma S_{b}(x, \omega)}+\sigma \int_{0}^{S_{b}(x, \omega)} e^{-\sigma r} F[\tilde{u}(x+r \omega)] d r
$$

or

$$
\left\{\begin{array}{l}
A u(x, \omega)=0, \\
u(x, \omega)=1 \quad \text { if }(x, \omega) \in \partial G .
\end{array}\right.
$$


Furthermore, it can be proved easily, as in $[10]$, that $q$ is the smallest solution among all solutions of (2.1) satisfying $0 \leq u(x, \omega) \leq 1$.

We can prove also the following lemma as in [10].

Lemma 2.1. $P_{(x, \omega)}\left[Z_{t} \rightarrow 0\right.$ or $Z_{t} \rightarrow \infty$ when $\left.t \rightarrow \infty\right]=1$ for every $(x, \omega) \in G$.

Let $E(x, y ; \lambda)$ be the function $\sigma e^{-(\sigma+\lambda)|x-y|} / 4 \pi|x-y|^{2}$ on $D \times D$ for each $\lambda$ and $c^{*}$ be the largest positive eigenvalue of the integral operator on $\mathbf{C}(D)$ induced by the kernel $E(x, y ; 0)$. Equation (2.2) has always the trivial solution $u(x, \omega) \equiv 1$. Therefore, the extinction problem is equivalent to that of the uniqueness of the solution for equation (2.2).

Lemma 2.2 (Pazy-Rabinowitz $[8]$ ). If $F^{\prime}[1] \leqq c^{*}$, then (1.6) has no non-trivial solution and hence $q(x, \omega) \equiv 1$. On the other hand if $F^{\prime}[1]>c^{*}$ then it has the unique non trivial solution which is, therefore, equal to $q(x, \omega)$. Furthermore, $q(x, \omega)<1$ if $(x, \omega) \in G-\partial G$ and $\inf _{(x, \omega)} q(x, \omega)>0$.

We shall say that the process is subcritical, critical, or supercritical according as $F^{\prime}[1]<c^{*}, F^{\prime}[1]=c^{*}$, or $F^{\prime}[1]>c^{*}$, respectively.

If we set

$$
M_{t} f(x, \omega)=E_{(x, \omega)}\left[\check{f}\left(X_{t}\right)\right],
$$

then the following lemma holds.

Lemma 2.3. There exist positive constants $T_{0}$ and $\rho$ such that for every $t \geq T_{0}$, and for every $f \in \mathbf{C}(G)$

$$
\left\|M_{t} f(x, \omega)-e^{\alpha t}\left(f, \psi^{*}\right) \psi(x, \omega)\right\| \leqq e^{\alpha t} O\left(e^{-\rho t}\|f\|\right)
$$

where $\alpha$ is the eigenvalue of $B$ with the maximal real part and $\phi\left(\psi^{*}\right)$ is the corresponding eigenfunction of $B$ (resp. $\left.B^{*}\right)$. When $f$ is in $\mathfrak{S}(G)$, then the same estimate holds if we replace only $\|f\|$ in the right hand side by $\|f\|_{2}$.

In addition, $\alpha$ is real and simple. The process is subcritical, critical or supercritical according as $\alpha<0, \alpha=0$ or $\alpha>0$, cf. $[6] . \psi$ is obtained 
by the following equation from the eigenfunction $\varphi \in \mathbb{C}(D)$ corresponding to the largest eigenvalue of the operator induced by $E(x, y ; \alpha)$,

$$
\psi(x, \omega)=\sigma \int_{0}^{S_{b}(x, \omega)} e^{-\sigma r} \varphi(x+r \omega) d r .
$$

By the theorem of Krein and Rutman [5], $\varphi$ is uniformly positive, therefore $\psi$ is positive on $G-\partial G$ and zero on $\partial G$. Also $\psi^{*}(x, \omega)=$ $\phi(x,-\omega)$.

\section{§ 3. The Asymptotic Behaviour of $T_{t} \hat{g}(x, \omega), \mathbf{I}$ :}

Subcritical Case, i.e. $\left(\alpha<0\right.$, or $\left.F^{\prime}[1]<c^{*}\right)$

In this case, $q(x, \omega) \equiv 1$, i.e. $P_{(x, \omega)}\left(e_{\partial}<+\infty\right)=\lim _{t \rightarrow \infty} q(t, x, \omega) \equiv 1$. If we define $u(t, x, \omega)=1-T_{t} \hat{g}(x, \omega)$ for each $g \in \mathbf{C}_{1}^{+}(G)$, then it tends to 0 as $t \rightarrow \infty$, since $q(t, x, \omega) \leq T_{t} \hat{g}(x, \omega)$. We shall discuss the rate of convergence assuming $F^{\prime \prime}[1]<+\infty$.

Theorem 3.1. For every $g \in \mathbb{C}_{1}^{+}(G)$,

$$
1-T_{t} \hat{g}(x, \omega)=\Gamma(g) e^{\alpha t} \psi(x, \omega)+e^{\alpha t} O\left(e^{-\delta t}\right)
$$

where $\Gamma(g)$ is a positive constant depending on $g$, and $\delta$ is a positive constant

Proof. From the definition and the simple calculation $u(t, x, \omega)$ $=1-T_{t} \hat{g}(x, \omega)$ satisfies

$$
\begin{aligned}
& \frac{\partial u(t, x, \omega)}{\partial t}=\omega \cdot \nabla u(t, x, \omega)-\sigma u(t, x, \omega)+\sigma F^{\prime}[1] \tilde{u}(t, x) \\
& -\frac{\sigma}{2} \tilde{u}(t, x)^{2} F^{\prime \prime}[\xi(t, x)] \\
& u(0+, x, \omega)=1-g(x, \omega) \\
& u(t, x, \omega)=0 \quad \text { if }(x, \omega) \in \partial G
\end{aligned}
$$

where

$$
1-\tilde{u}(t, x) \leq \xi(t, x) \leq 1 .
$$

Using Duhamel's principle, we obtain 
(3.3) $u(t, x, \omega)=M_{t}(1-g)(x, \omega)-\frac{\sigma}{2} \int_{0}^{t} M_{t-s}\left\{F^{\prime \prime}[\xi(s)] \tilde{u}(s)^{2}\right\}(x, \omega) d s$.

Combining this with (2.4)

$$
\begin{gathered}
u(t, x, \omega)=e^{\alpha t}\left(1-g, \psi^{*}\right) \psi(x, \omega)+e^{\alpha t} O\left(e^{-\rho t}\right) \\
-\frac{\sigma}{2} \int_{0}^{t} M_{t-s}\left\{F^{\prime \prime}[\xi(s)] \tilde{u}(s)^{2}\right\}(x, \omega) d s
\end{gathered}
$$

for every $t \geq T_{0}$. Since the third term of (3.4) is non-positive, there exists a positive constant $K$ such that

$$
\begin{aligned}
& u(t, x, \omega) \leq K \cdot e^{\alpha t} \text { for every } t \geq 0, \text { and therefore } \\
& \tilde{u}(t, x) \leq K \cdot e^{\alpha t} \quad \text { for every } t \geq 0 .
\end{aligned}
$$

Now, let

$$
\int_{0}^{t} M_{t-s}\left\{F^{\prime \prime}[\xi(s)] \tilde{u}(s)^{2}\right\}(x, \omega) d s=\int_{0}^{(1-\varepsilon) t}+\int_{(1-\varepsilon) t}^{t} \equiv I_{1}+I_{2}
$$

where $\varepsilon$ is a constant satisfying $0<\varepsilon<\frac{1}{2}$. Then from Lemma 2.3, if $t \varepsilon \geq T_{0}$

$$
\begin{gathered}
I_{1}=\psi(x, \omega) \int_{0}^{(1-\varepsilon) t} d s e^{\alpha(t-s)}\left\{\int_{G} F^{\prime \prime}[\xi(s, y)] \tilde{u}(s, y)^{2} \psi^{*}(y, \mu) d y d \mu\right\} \\
+\int_{0}^{(1-\varepsilon) t} e^{\alpha(t-s)} O\left(e^{-\rho(t-s)}\left\|\tilde{u}(s, \cdot)^{2}\right\|\right) d s .
\end{gathered}
$$

Noting $\left\|u(s, \cdot)^{2}\right\| \leq K e^{\alpha t}$ and assuming $\alpha+\rho<0$,

$$
\begin{aligned}
I_{1}= & e^{\alpha t} \psi(x, \omega) \int_{0}^{\infty} e^{-\alpha s} d s\left\{\int_{G} F^{\prime \prime}[\xi(s, y)] \tilde{u}(s, y)^{2} \psi^{*}(y, \mu) d y d \mu\right\} \\
& -e^{\alpha t} \psi(x, \omega) \int_{(1-\varepsilon) t}^{\infty} e^{-\alpha s} d s\left\{\int_{G} F^{\prime \prime}[\xi(s, y)] \tilde{u}(s, y)^{2} \psi^{*}(y, \mu) d y d \mu\right\} \\
& +e^{\alpha t} \int_{0}^{(1-\varepsilon) t} O\left(e^{-\alpha s-\rho(t-s)}\|\tilde{u}(s, \cdot)\|^{2}\right) d s \\
= & e^{\alpha t} \psi(x, \omega) \int_{0}^{\infty} e^{-\alpha s} d s\left\{\int_{G} F^{\prime \prime}[\xi(s, y)] \tilde{u}(s, y)^{2} \psi^{*}(y, \mu) d y d \mu\right\} \\
& +e^{\alpha t} \psi(x, \omega) O\left(e^{\alpha(1-\varepsilon) t}\right)+e^{\alpha t}\left(e^{-\rho t}\right),
\end{aligned}
$$

and 


$$
\begin{aligned}
I_{2} & =\int_{(1-\varepsilon) t}^{t} M_{t-s}\left\{F^{\prime \prime}[\xi(s)] \tilde{u}(s)^{2}\right\}(x, \omega) d s \leq \int_{(1-\varepsilon) t}^{t} F^{\prime \prime}[1] K^{2} M_{t-s}\left(e^{2 \alpha s}\right)(x, \omega) d s \\
& \leq F^{\prime \prime}[1] K^{2} c \int_{(1-\varepsilon) t}^{t} e^{\alpha(t-s)} e^{2 \alpha s} d s=e^{\alpha t} O\left(e^{\alpha(1-\varepsilon) t}\right)
\end{aligned}
$$

where $c$ is a positive constant. Therefore,

$$
\begin{gathered}
u(t, x, \omega)=e^{\alpha t} \psi(x, \omega) \int_{G} \psi^{*}(y, \mu)\left\{1-g(y, \mu)-\frac{\sigma}{2} \int_{0}^{\infty} F^{\prime \prime}[\xi(s, y)]\right. \\
\left.\quad \times \tilde{u}(s, y)^{2} e^{-\alpha s} d s\right\} d y d \mu+e^{\alpha t} O\left(e^{-\delta t}\right) \\
\left(\equiv \Gamma(g) e^{\alpha t} \psi(x, \omega)+e^{\alpha t} O\left(e^{-\delta t}\right)\right)
\end{gathered}
$$

for some positive constant $\delta$, and the theorem is proved if we show that $\Gamma(g)$ is positive. Since the left hand side of (3.9) is non negative, $\Gamma(g)$ $\geq 0$, and it is sufficient to check that $\Gamma(g) \neq 0$. We can prove the following Lemma in a similar method as above using the inequality $1-e^{-f / t} \leq f / t$.

Lemma 3.1. For every $f \in \mathbf{C}_{1}^{+}(G)$

$$
1-T_{s}\left(\widehat{e^{-f i t}}\right)(x, \omega)=\psi(x, \omega)\left(f, \psi^{*}\right) \frac{e^{\alpha s}}{t}+e^{\alpha s} O\left(\frac{1}{t^{2}}\right) .
$$

New let $f$ be a function in $\mathbf{C}_{1}^{+}(G)$ not identically zero. Then, for every $g \in \mathbf{C}_{1}^{+}(G)$, if $t$ is sufficiently large,

$$
1-T_{t} \hat{g}(x, \omega) \geq 1-T_{t}\left(e^{-f / t}\right)(x, \omega)=\psi(x, \omega)\left(f, \psi^{*}\right) \frac{e^{\alpha t}}{t}+e^{\alpha t} O\left(\frac{1}{t^{2}}\right) .
$$

Therefore it is impossible to have $\Gamma(g)=0$ and the proof is complete.

\section{§ 4. The Asymptotic Behaviour of $\left.\widehat{T_{t}} \widehat{\left(e^{-g} / t\right.}\right)$ : \\ Critical Case $\left(\alpha=0\right.$, or $\left.F^{\prime}[1]=c^{*}\right)$}

In this case, the extinction probability $q(x, \omega)$ is also identical to 1 as in the sub-critical case. But Lemma 2.3 shows that the expected number of neutrons does not tend to zero as $t \rightarrow \infty$. We shall study the asymptotic behaviour of $T_{t}\left(\widehat{e^{-g / t}}\right)$ as $t \rightarrow \infty$, assuming that $F^{\prime \prime \prime}[1]<+\infty$. 
Theorem 4.1. There exists a positive constant $a>0$ such that for every $g \in \mathbb{C}_{1}^{+}(G)$ satisfying $\|g\| \leq a$,

$$
\lim _{t \rightarrow \infty} \frac{1-T_{t}\left(\widehat{e^{-g / t}}\right)(x, \omega)}{1 / t}=\frac{\psi(x, \omega)\left(g, \psi^{*}\right)}{1+\frac{2}{\sigma} F^{\prime \prime}[1]\left(\varphi^{2}, \psi^{*}\right)\left(g, \psi^{*}\right)}
$$

where $\varphi(x)=\tilde{\psi}(x)$.

Proof. For $g \in \mathbf{C}_{1}^{+}(G)$, let $u(t, s ; x, \omega)$ be

$$
u(t, s: x, \omega)=1-T_{s}\left(\widehat{e^{-g / t}}\right)(x, \omega) \quad s \leq t .
$$

Then

$$
u(t, s: x, \omega)=M_{s}\left(1-e^{-g / t}\right)(x, \omega)-\int_{0}^{s} M_{s-v}\left\{\frac{\sigma}{2} F^{\prime \prime}[\xi(v)] \tilde{u}(t, v)^{2}(x, \omega) d v\right\}
$$

where $\tilde{u}(t, v: x)=\frac{1}{4 \pi} \int_{\Omega} u(t, v: x, \omega) d \omega$ and $1-\tilde{u}(t, v, \lambda) \leqq \xi(v) \leqq 1$.

Since $1-e^{-g / t} \leq g / t$, we have

$$
u(t, s: x, \omega) \leq M_{s}\left(1-e^{-g / t}\right)(x, \omega) \leq \frac{k_{1}}{t}
$$

where $k_{1}$ is some positive constant independent of $g$.

For the proof of theorem, it is sufficient to show the existence of the constants $a$ and $b, 0<b<1$, such that, for $g \in \mathbf{C}_{1}^{+}$and $\|g\| \leq a$, then for each $n(n=1,2, \ldots)$,

$$
\begin{gathered}
u(t, s: x, \omega)=J_{1}^{(n)}(s)+J_{2}^{(n)}(s)+J_{3}^{(n)}(s), \\
J_{1}^{(n)}(s)=\psi(x, \omega) \sum_{i=1}^{n}(-1)^{i-1} Q^{i-1} s^{i-1}\left(g, \psi^{*}\right)^{i} / t^{i} \\
\left|J_{2}^{(n)}(s)\right| \leq b^{n+1} / t \\
\lim _{s \rightarrow \infty}\left[\sup _{\substack{t \\
s \leq t}} t \cdot\left|J_{3}^{(n)}(s)\right|\right]=0
\end{gathered}
$$

where $Q=\frac{\sigma}{2} F^{\prime \prime}[1]\left(\varphi^{2}, \phi^{*}\right)$.

For the convenience of the proof, we introduce the notation $f(t, s$; $x, \omega)=o_{s}(1)(t \geq s,(x, \omega) \in G)$ which means that $\lim _{s \rightarrow \infty}\left\{\sup _{t: t \geq s,(x, \omega)}|f(t, s ; x, \omega)|\right\}$ $=0$. Using this notation, (4.5.3) is written as $t \cdot J_{3}^{(i)}(s) \stackrel{t i t s,(x, \omega)}{=} o_{s}(1)$.

We shall prove (4.5) by mathematical induction. For $n=1$, by (4.4) 
and the assumption that $F^{\prime \prime}[1]<+\infty$,

$$
\begin{aligned}
u(t, s: x, \omega)= & M_{s}\left(1-e^{-g / t}\right)(x, \omega) \\
& -\int_{0}^{s} M_{s-v}\left\{\frac{\sigma}{2} F^{\prime \prime}[1] \tilde{u}(t, v)^{2}(1+O(\tilde{u}(t, v))(x, \omega) d v\}\right.
\end{aligned}
$$

Since $1-e^{-g / t}=g / t+O\left(\frac{1}{t^{2}}-\right)$, we have

$$
\begin{aligned}
u(t, s: x, \omega)=\psi & (x, \omega) \frac{\left(g, \psi^{*}\right)}{t}+O\left(\frac{1}{t^{2}}\right) \\
& \quad-\int_{0}^{s} M_{s-v}\left\{\frac { \sigma } { 2 } F ^ { \prime \prime } \left[1\left[\tilde{u}(t, v)^{2}\right\}(x, \omega) d v+s O\left(\frac{1}{t^{3}}\right) .\right.\right.
\end{aligned}
$$

Let

$$
J_{1}^{(1)}(s)=\psi(x, \omega)\left(g, \psi^{*}\right) / t
$$

and

$$
J_{2}^{(1)}(s)=-\int_{0}^{s} M_{s-v}\left\{\frac{\sigma}{2} F^{\prime \prime}[1] \tilde{u}(t, v)^{2}\right\}(x, \omega) d v .
$$

Then,

$$
\left|J_{2}^{(1)}(s)\right| \leqq \frac{s}{t^{2}} k_{1}^{2} k_{2}\|g\|^{2} \leqq \frac{k_{1}^{2} k_{2}\|g\|^{2}}{t}
$$

where

$$
k_{2}=\sup _{s, x, \omega} M_{s}\left(\frac{\sigma}{2} F^{\prime \prime}(1)\right)(x, \omega)<+\infty .
$$

Taking $k_{3}$ such that $k_{1}^{2} k_{2}<k_{3}^{2}, k_{3}>\max \left(k_{2}, 1\right)$, the case $n=1$ is true if we take $a, b$ such that $a \leqq 1 / k_{3}^{2}$ and $b=k_{3}^{-1}$.

Now, assume that (4.5) holds for $n$. From (4.7),

$$
\text { (4.9) } \begin{aligned}
u(t, s: x, \omega)=\psi(x, \omega) \frac{\left(g, \phi^{*}\right)}{t} & -\int_{0}^{s} M_{s-v}\left\{\frac{\sigma}{2} F^{\prime \prime}[1] \tilde{u}(t, v)^{2}\right\}(x, \omega) d v \\
& +\frac{o_{s}(1)}{t},
\end{aligned}
$$

and

$$
\int_{0}^{s} M_{s-v}\left\{\frac{\sigma}{2} F^{\prime \prime}[1] \tilde{u}(t, v)^{2}\right\}(x, \omega) d v
$$




$$
\begin{aligned}
& =\int_{0}^{s} M_{s-v}\left\{\frac{\sigma}{2} F^{\prime \prime}[1]\left(\tilde{J}_{1}^{(n)}(v)+\tilde{J}_{2}^{(n)}(v)+\tilde{J}_{3}^{(n)}(v)\right)^{2}\right\}(x, \omega) d v \\
& =\int_{0}^{s} M_{s-v}\left\{\frac{\sigma}{2} F^{\prime \prime}[1]\left(\tilde{J}_{1}^{(n)}(v)\right)^{2}\right\}(x, \omega) d v \\
& +2 \int_{0}^{s} M_{s-v}\left\{\frac{\sigma}{2} F^{\prime \prime}[1] \tilde{J}_{1}^{(n)}(v) \tilde{J}_{2}^{(n)}(v)\right\}(x, \omega) d v \\
& +\int_{0}^{s} M_{s-v}\left\{\frac{\sigma}{2} F^{\prime \prime}[1]\left(\tilde{J}_{2}^{(n)}\right)(v)^{2}\right\}(x, \omega) d v \\
& +\int_{0}^{s} M_{s-v}\left\{\frac{\sigma}{2} F^{\prime \prime}[1] \tilde{J}_{3}^{(n)}(v) \tilde{u}(t, v)\right\}(x, \omega) d v \\
& +\int_{0}^{s} M_{s-v}\left\{\frac{\sigma}{2} F^{\prime \prime}[1] \tilde{J}_{3}^{(n)}(v)\left(\tilde{J}_{1}^{(n)}(v)+\tilde{J}_{2}^{(n)}(v)\right)\right\}(x, \omega) d v
\end{aligned}
$$

where, $\tilde{J}_{i}^{(n)}(v)=\frac{1}{4 \pi} \int_{\Omega} J_{i}^{(n)}(v) d \omega$. By the definition

$$
\tilde{J}_{1}^{(n)}(v)=\varphi(x) \cdot \sum_{i=1}^{n}(-1)^{i-1} Q^{i-1} v^{i-1} \frac{\left(g, \phi^{*}\right)^{i}}{t^{i}}
$$

and $\tilde{J}_{2}^{(n)}(v)\left(\tilde{J}_{3}^{(n)}(v)\right)$ satisfies (4.5.2) (resp. (4.5.3)). $\quad\left\{\tilde{J}_{1}^{(n)}(v)\right\}^{2}$ can be written as

$$
\left\{\tilde{J}_{1}^{(n)}(v)\right\}^{2}=\varphi(x)^{2}\left\{\sum_{i=1}^{n}(-1)^{i-1} Q^{i-1} v^{i-1}\left(g, \psi^{*}\right)^{i} / t^{i}\right\}^{2}=\left(I_{1}+I_{2}\right) \varphi(x)^{2}
$$

where

$$
I_{1}=\sum_{i=2}^{n+1}(i-1)(-1)^{i} Q^{i-2}\left(g, \psi^{*}\right)^{i} v^{i-2} / t^{i}
$$

and

$$
I_{2}=\sum_{\substack{1 \leq k, l \leq n \\ k+l>n+1}}(-1)^{k+l} Q^{k+l-2}\left(g, \psi^{*}\right)^{k+l} v^{k+l-2} / t^{k+l-2} .
$$

If we can prove

$$
\text { (4.12) } \int_{0}^{s} M_{s-v}\left\{\frac{\sigma}{2} F^{\prime \prime}[1] \varphi^{2} v^{k-2}\right\}(x, \omega) d v=\frac{Q s^{k-1}}{k-1} \psi(x, \omega)+s^{k-1} o_{s}(1)
$$

then, we have 
(4.13) $\int_{0}^{s} M_{s-v}\left\{\frac{\sigma}{2} F^{\prime \prime}[1] I_{1} \varphi^{2}\right\}(x, \omega) d v=\psi(x, \omega) \sum_{i=2}^{n+1}(-1)^{i} Q^{i-1} \frac{s^{i-1}}{t^{i}}\left(g, \psi^{*}\right)^{i}$

$$
+\frac{o_{s}(1)}{t}
$$

To prove (4.12), let $\varepsilon$ be an arbitrary small positive number. Then, there exists a positive constant $T$ such that, for every $t \geqq T$,

$$
Q \cdot \psi(x, \omega)-\varepsilon \leqq M_{t}\left\{\frac{\sigma}{2} F^{\prime \prime}[1] \varphi^{2}\right\}(x, \omega) \leqq Q \cdot \psi(x, \omega)+\varepsilon .
$$

Therefore,

$$
\int_{s-T}^{s} M_{s-v}\left\{\frac{\sigma}{2} F^{\prime \prime}[1] \varphi^{2} v^{k-2}\right\} d v \leq k_{4} \int_{s-T}^{s} v^{k-2} d v=s^{k-1} o_{s}(1)
$$

where

$$
k_{4}=\sup _{s, x, \omega} M_{s}\left\{\frac{\sigma}{2} F^{\prime \prime}[1] \varphi^{2}\right\}(x, \omega)<+\infty
$$

and

$$
\begin{aligned}
\{Q \cdot \psi(x, \omega)-\varepsilon\} \int_{0}^{s-T} v^{k-2} d v & \leqq \int_{0}^{s-T} M_{s-v}\left\{\frac{\sigma}{2} F^{\prime \prime}[1] \varphi^{2} v^{k-2}\right\}(x, \omega) d v \\
& \leq\{Q \cdot \psi(x, \omega)+\varepsilon\} \int_{0}^{s-T} v^{k-2} d v .
\end{aligned}
$$

If we set

$$
I(s)=\int_{0}^{s-T} M_{s-v}\left\{\frac{\sigma}{2} F^{\prime \prime}[1] \varphi^{2} v^{k-2}\right\}(x, \omega) d v
$$

then

$$
\frac{Q \cdot \psi(x, \omega)-\varepsilon}{k-1} \leqq \lim _{s \rightarrow \infty} \frac{I(s)}{(s-T)^{k-1}} \leqq \varlimsup_{s \rightarrow \infty} \frac{I(s)}{(s-T)^{k-1}} \leqq \frac{Q \cdot \psi(x, \omega)+\varepsilon}{k-1}
$$

and the estimate (4.12) is proved.

Since,

$$
\begin{aligned}
\left|I_{2}\right| \leq & (n-1) Q^{n}\left(g, \psi^{*}\right)^{n+2} \frac{v^{n}}{t^{n+2}}+(n-2) Q^{n+1}\left(g, \psi^{*}\right)^{n+3} \frac{v^{n+1}}{t^{n+3}} \\
& +\cdots+Q^{2 n-2}\left(g, \psi^{*}\right)^{2 n} \frac{v^{2 n-2}}{t^{2 n}}
\end{aligned}
$$


we have

$$
\begin{aligned}
& \left|\int_{0}^{s} M_{s-v}\left\{\frac{\sigma}{2} F^{\prime \prime}[1] I_{2} \varphi^{2}\right\}(x, \omega) d v\right| \\
& \leqq Q^{n}\left(g, \phi^{*}\right)^{n+2} k_{4}\left\{\frac{(n-1)}{t^{n+2}} \int_{0}^{s} v^{n} d v+\frac{(n-2)}{t^{n+3}} Q \cdot\left(g, \psi^{*}\right) \int_{0}^{s} v^{n+1} d v\right. \\
& \left.\quad+\cdots+\frac{Q^{n-2}\left(g, \psi^{*}\right)^{n-2}}{t^{2 n}} \int_{0}^{s} v^{2 n-2} d v\right\} \\
& \leqq \\
& \frac{Q^{n}\left(g, \psi^{*}\right)^{n+2}}{t} \cdot \frac{k_{4}}{1-Q\left(g, \psi^{*}\right)} .
\end{aligned}
$$

For the second term of $(4.10)$, since $\left|\tilde{J}_{1}^{(n)}(v)\right| \leqq \frac{\varphi(x) \cdot\left(g, \psi^{*}\right)}{t}$

$$
\begin{aligned}
& \times \frac{1}{1-Q\left(g, \psi^{*}\right),} \text { we have } \\
& \quad\left|\int_{0}^{s} M_{s-v}\left\{\frac{\sigma}{2} F^{\prime \prime}[1] \tilde{J}_{1}^{(n)}(v) \tilde{J}_{2}^{(n)}(v)\right\}(x, \omega) d v\right| \\
& \quad \leq \frac{\left(g, \psi^{*}\right) b^{n+1}}{t^{2}\left(1-Q\left(g, \psi^{*}\right)\right)} \int_{0}^{s} M_{s-v}\left\{\frac{\sigma}{2} F^{\prime \prime}[1] \varphi^{2}\right\}(x, \omega) d v \\
& \quad \leqq \frac{\left(g, \psi^{*}\right) b^{n+1} k_{4}}{t\left(1-Q\left(g, \psi^{*}\right)\right)} .
\end{aligned}
$$

For the third term, we have

$$
\left|\int_{0}^{s} M_{s-v}\left\{\frac{\sigma}{2} F^{\prime \prime}[1]\left(\tilde{J}_{2}^{(n)}(v)\right)^{2}\right\}(x, \omega) d v\right| \leq \frac{s}{t^{2}} b^{2(n+1)} \cdot k_{2} .
$$

For the fourth term, we have

$$
\begin{aligned}
& \left|\int_{0}^{s} M_{s-v}\left\{\frac{\sigma}{2} F^{\prime \prime}[1] \tilde{J}_{3}^{(n)}(v) \tilde{u}(t, v)\right\}(x, \omega) d v\right| \\
& \leqq \frac{k_{1}\|g\|}{t} \int_{0}^{s} M_{s-v}\left\{\frac{\sigma}{2} F^{\prime \prime}[1]\left|\tilde{J}_{3}^{(n)}(v)\right|\right\}(x, \omega) d v \\
& \leqq \frac{k_{1} k_{2}\|g\|}{t^{2}} \int_{0}^{s} o_{v}(1) d v=\frac{o_{s}(1)}{t} .
\end{aligned}
$$

And, for the last term, we have 


$$
\begin{aligned}
& \left|\int_{0}^{s} M_{s-v}\left\{\frac{\sigma}{2} F^{\prime \prime}[1] \tilde{J}_{3}^{(n)}(v)\left(\tilde{J}_{1}^{(n)}(v)+\tilde{J}_{2}^{(n)}(v)\right)\right\}(x, \omega) d v\right| \\
& \leqq \int_{0}^{s} M_{s-v}\left\{\frac{\sigma}{2} F^{\prime \prime}[1]\left|\tilde{J}_{3}^{(n)}(v)\right|\left|\tilde{J}_{1}^{(n)}(v)\right|\right\}(x, \omega) d v \\
& \quad+\int_{0}^{s} M_{s-v}\left\{\frac{\sigma}{2} F^{\prime \prime}[1]\left|\tilde{J}_{2}^{(n)}(v)\right|\left|\tilde{J}_{3}^{(n)}(v)\right|\right\}(x, \omega) d v \\
& \leqq \frac{k_{2} \varphi(x)\left(g, \psi^{*}\right)}{t\left(1-Q\left(g, \psi^{*}\right)\right)} \int_{0}^{s} \frac{o_{v}(1)}{t} d v+\frac{k_{2} b^{n+1}}{t} \int_{0}^{s} \frac{o_{v}(1)}{t} d v=\frac{o_{s}(1)}{t} .
\end{aligned}
$$

If we set

$$
\begin{aligned}
J_{2}^{(n+1)}(s)= & -\int_{0}^{s} M_{s-v}\left\{\frac{\sigma}{2} F^{\prime \prime}[1] I_{2} \varphi^{2}\right\}(x, \omega) d v \\
& -2 \int_{0}^{s} M_{s-v}\left\{\frac{\sigma}{2} F^{\prime \prime}[1] \tilde{J}_{1}^{(n)}(v) \tilde{J}_{2}^{(n)}(v)\right\}(x, \omega) d v \\
& -\int_{0}^{s} M_{s-v}\left\{\frac{\sigma}{2} F^{\prime \prime}[1]\left(\tilde{J}_{2}^{(n)}(v)\right)^{2}\right\}(x, \omega) d v
\end{aligned}
$$

then

$$
\left|J_{2}^{(n+1)}(s)\right| \leq \frac{b^{n+1}}{t}\left\{\frac{k_{4} \cdot Q^{n}\left(g, \psi^{*}\right)^{n+2}}{b^{n+1}\left(1-Q\left(g, \psi^{*}\right)\right)}+\frac{2 \cdot k_{4}\left(g, \psi^{*}\right)}{1-Q\left(g, \psi^{*}\right)}+K_{2} b^{n+1}\right\}
$$

Now take $a>0$ such that the bracket in the right hand side of (4.15) is less than $b$ and $\left|Q\left(g, \psi^{*}\right)\right|<1$ for every $\|g\| \leqq a$. (We can determine such $a$ independent of $n$.)

Then, for every $g,\|g\| \leqq a$, we have $\left|J_{2}^{(n+1)}\right| \leqq \frac{b^{n+2}}{t}$. So (4.5) is completely proved.

\section{§ 5. The Asymptotic Behaviour of $T_{t} \hat{g}(x, \omega)$ As $t \rightarrow \infty$, II:}

\section{Critical Case}

In this section we shall study the asymptotic behaviour of $T_{t} \hat{g}(x, \omega)$ assuming that $F^{\prime \prime \prime}[1]<+\infty$.

Theorem 5.1. For every $g \in \mathbf{C}_{1}^{+}(G)$ and $\|g\|<1, u(t, x, \omega)=$ 
$1-T_{t} \hat{g}(x, \omega)$ satisfies

$$
u(t, x, \omega)=\frac{\psi(x, \omega)}{\int_{G} \frac{\sigma}{2} F^{\prime \prime}[1] \varphi^{2} \phi^{*} d x d \omega} \cdot \frac{1}{t}+O\left(t^{\varepsilon-2}\right)
$$

for every $\varepsilon>0$.

Proof. Let $\varepsilon$ be a fixed arbitrarily small positive number, and $f(t)$ be,

$$
f(t)=\int_{G} u(t, x, \omega) \psi^{*}(x, \omega) d x d \omega .
$$

We shall first show that there exist positive constants $k_{1}, k_{2}, T_{1}$ and $T_{2}$, such that, for every $t \geq T_{1}$,

$$
f(t) \leqq k_{1} t^{\varepsilon-1}
$$

and for every $t \geq T_{2}$,

$$
u(t, x, \omega) \leqq k_{2} t^{\varepsilon-1} .
$$

By the definition, $f(t)$ satisfies

$$
\frac{d f(t)}{d t}=-\frac{\sigma}{2} \int_{G} F^{\prime \prime}[\xi(t, x)] \tilde{u}(t, x)^{2} \psi^{*}(x, \omega) d x d \omega
$$

where $1-\tilde{u}(t, x) \leq \xi(t, x) \leq 1$. Since $\tilde{u}(t, x)$ is a continuous function of $x$ and $\tilde{u}(t, x) \downarrow 0$ as $t \rightarrow \infty$, by Dini's theorem, there exist positive constants $T_{3}$ and $\eta<1$, such that, for every $t \geqq T_{3}, \eta \leqq 1-\widetilde{u}(t, x) \leqq \xi(t, x)$ $\leqq 1$.

From the definition of $\psi^{*}$, it follows that

$$
\varphi(x)=\frac{1}{4 \pi} \int_{\Omega} \psi(x, \omega) d \omega=\frac{1}{4 \pi} \int_{\Omega} \psi^{*}(x, \omega) d \omega .
$$

Then

$$
\frac{d f(t)}{d t} \leqq-\frac{\sigma F^{\prime \prime}[\eta] c_{1}}{2} \int_{D} \tilde{u}(t, x)^{2} d x
$$

where, $c_{1}$ is a positive constant such that $c_{1} \leqq \varphi(x)$. Using the Hölder's inequality, we have for any $\varepsilon^{\prime}>0$,

$$
f(t) \leqq m(D)^{\varepsilon^{\prime} / 1+\varepsilon^{\prime}}\left[\int_{D} d x\left\{\frac{1}{4 \pi} \int_{\Omega} u(t, x, \omega) \psi^{*}(x, \omega) d \omega\right\}^{1+\varepsilon^{\prime}}\right] \frac{1}{1+\varepsilon^{\prime}},
$$


where $m(D)$ is the Lebesgue measure of the domain $D$. Therefore,

$$
\begin{aligned}
f(t)^{1+\varepsilon^{\prime}} & \leqq m(D)^{\varepsilon^{\prime}} \int_{D} d x\left\{\frac{1}{4 \pi} \int_{\Omega} u(t, x, \omega) \psi^{*}(x, \omega) d \omega\right\}^{1+\varepsilon^{\prime}} \\
& \leqq m(D)^{\varepsilon^{\prime}} \int_{D} d x\left\{\frac{1}{4 \pi} \int_{\Omega} u(t, x, \omega)^{1+\varepsilon^{\prime}} d \omega\right\}\left\{\int_{\Omega} \psi^{*}(x, \omega)^{\frac{1+\varepsilon^{\prime}}{\varepsilon^{\prime}}} d \omega\right\}^{\varepsilon^{\prime}} \\
& \leqq c_{3} \int_{D} \tilde{u}(t, x) d x \leqq C_{4}\left(\int_{D} \tilde{u}^{2}(t, x) d x\right)^{\frac{1}{2}}
\end{aligned}
$$

for some positive constant $c_{4}$, since $0 \leqq u(t, x, \omega) \leqq 1$. Hence, we have

$$
\frac{d f}{d t} \cong-c_{5} f(t)^{2+2 \varepsilon^{\prime}}
$$

and

$$
\frac{d}{d t}\left(1 / f(t)^{1+2 \varepsilon^{\prime}}\right) \geqq c_{6}
$$

where $c_{6}$ is some positive constant. Integrating (5.8), we get, for some positive constant $c_{7}$,

$$
\frac{1}{f(t)^{1+2 \varepsilon^{\prime}}} \geqq c_{7} t
$$

and (5.3) is proved. (5.4) is obvious from the following:

$$
\begin{aligned}
u(2 t, x, \omega) & =\left(M_{t} u(t)\right)(x, \omega)-\int_{0}^{t} M_{t-s}\left\{\frac{\sigma}{2} F^{\prime \prime}[\xi(s+t)] \tilde{u}(s+t)^{2}\right\}(x, \omega) d s \\
& \leqq\left(M_{t} u(t)\right)(x, \omega) \\
& =\phi(x, \omega) \int_{G} u(t, y, \mu) \psi^{*}(y, \mu) d y d \mu+O\left(e^{-\beta t}\right) \\
& =\phi(x, \omega) f(t)+O\left(e^{-\rho t}\right) .
\end{aligned}
$$

We need, further, the following two lemmas.

Lemma 5.1. For erery $\varepsilon \geqq 0, L \geqq 1$ and $\rho>0$,

$$
\int_{L}^{t} \frac{e^{-\rho(t-s)}}{s^{2-\varepsilon}} d s=O\left(t^{-2+\varepsilon}\right)
$$


Lemma 5.2. For every $\varepsilon>0$, if

$$
g(t)=k \int_{t}^{\infty} g(s)^{2} d s\left(1+O\left(t^{\varepsilon-1}\right)\right) .
$$

then

$$
g(t)=\frac{1}{k t}\left(1+O\left(t^{\varepsilon-1}\right)\right) .
$$

Proof. Set $v(t)=k \int_{t}^{\infty} g(s)^{2} d s$, then

$$
v(t)=g(t)\left(1+O\left(t^{\varepsilon-1}\right)\right)
$$

and

$$
\frac{d v(t)}{d t}=-k g(t)^{2}=-k v(t)^{2}\left(1+O\left(t^{\varepsilon-1}\right)\right) .
$$

or

$$
\frac{d}{d t}\left(\frac{1}{v(t)}\right)=k\left(1+O\left(t^{\varepsilon-1}\right)\right) .
$$

If we fix $T$ sufficiently large,

$$
\begin{aligned}
& \frac{1}{v(t)}=k \int_{T}^{t}\left(1+O\left(s^{\varepsilon-1}\right)\right) d s+\frac{1}{v(T)} \\
& \quad=k t\left\{1+O\left(\frac{1}{t}\right)+\frac{1}{t} \int_{T}^{t} O\left(s^{\varepsilon-1}\right) d s\right\} \\
& =k t\left\{1+O\left(t^{\varepsilon-1}\right)\right\},
\end{aligned}
$$

and the lemma is proved.

Now let us return to the proof of the theorem. In the sequel, every $k_{i}\left(\right.$ or $\left.T_{i}\right)$ is some positive constant.

By the definition,

$$
u(t, x, \omega)=M_{t}(1-g)(x, \omega)-\int_{0}^{t} M_{t-s}\left\{\frac{\sigma}{2} F^{\prime \prime}[\xi(s)] \tilde{u}(s)^{2}\right\}(x, \omega) d s .
$$

From Lemma 2.3 , for every $t \geqq T_{0}$,

$$
M_{t}(1-g)(x, \omega)=\psi(x, \omega) \int_{G}\{1-g(y, \mu)\} \psi^{*}(y, \mu) d y d \mu+O\left(e^{-\rho t}\right) .
$$

For fixed $T_{4}$, which is larger than $T_{0}$ and $T_{2}$,

$$
\int_{0}^{t} M_{t-s}\left\{\frac{\sigma}{2} F^{\prime \prime}[\xi(s)] \tilde{u}(s)^{2}\right\}(x, \omega) d s
$$




$$
\begin{aligned}
= & \phi(x, \omega) \int_{0}^{t-T_{4}} d s\left\{\int_{G} \frac{\sigma}{2} F^{\prime \prime}[\xi(s, y)] \tilde{u}(x, y)^{2} \psi^{*}(y, \mu) d y d \mu\right\} \\
& +\int_{0}^{t-T_{4}} d s O\left(e^{-\rho(t-s)} \frac{\sigma}{2}\left\|F^{\prime \prime}[\xi(s)] \tilde{u}(s)^{2}\right\|\right) \\
& +\int_{t-T_{4}}^{t} M_{t-s}\left\{\frac{\sigma}{2} F^{\prime \prime}[\xi(s)] \tilde{u}(s)^{2}\right\}(x, \omega) d s .
\end{aligned}
$$

Let $I$ and $J$ be the second term and the third term respectively, then,

$$
\begin{aligned}
|I| \leqq & \int_{0}^{L}\left|O\left(e^{-\rho(t-s)} \frac{\sigma}{2}\left\|F^{\prime \prime}[\xi(s)] \tilde{u}(s)^{2}\right\|\right)\right| d s \\
& +\int_{L}^{t-T_{4}} \mid O\left(e^{-\rho(t-s)} \frac{\sigma}{2}\left\|F^{\prime \prime}[\xi(s)] \tilde{u}(s)^{2}\right\|\right) d s \\
& \leqq k_{3} e^{-\rho t}+k_{4} \int_{L}^{\infty} \frac{e^{-\rho(t-s)}}{s^{2-2 \varepsilon}} d s .
\end{aligned}
$$

It follows from Lemma 5.1 that $I=O\left(t^{2 \varepsilon-2}\right)$. On the other hand,

$$
|J|=k_{5} \int_{t-T_{4}}^{t} M_{t-s}\left(\frac{1}{s^{2-2 \varepsilon}}\right)(x, \omega) d s=O\left(t^{2 \varepsilon-2}\right) .
$$

Since $u(t, x, \omega) \downarrow 0$ as $t \rightarrow \infty$, we have from (5.11) and (5.12)

$$
\begin{aligned}
\psi(x, \omega) \int_{G}\{1-g(y, \mu)\} \psi^{*}(y, \mu) d y d \mu \\
\quad=\psi(x, \omega) \int_{0}^{\infty} d s\left\{\int_{G} \frac{\sigma}{2} F^{\prime \prime}[\xi(s, y)] \tilde{u}(s, y)^{2} \psi^{*}(y, \mu) d y d \mu\right\} \\
=\psi(x, \omega) \int_{0}^{\infty} h(s) d s
\end{aligned}
$$

where $h(s)$ is the function in the bracket \{\} . Therefore,

(5.15) $u(t, x, \omega)=\psi(x, \omega) \int_{0}^{\infty} h(s) d s-\int_{0}^{t} M_{t-s}\left\{\frac{\sigma}{2} F^{\prime \prime}[\xi(s)] \tilde{u}(s)^{2}\right\}(x, \omega) d s$

$$
\begin{aligned}
& +O\left(e^{-\rho t}\right) \\
= & \psi(x, \omega) \int_{t}^{\infty} h(s) d s \\
& +\int_{0}^{t-T_{4}} d s\left[\psi(x, \omega) h(s)-M_{t-s}\left\{\frac{\sigma}{2} F^{\prime \prime}[\xi(s)] \tilde{u}(s)^{2}\right\}(x, \omega)\right]
\end{aligned}
$$


Since

$$
\begin{aligned}
& +\int_{t-T_{4}}^{t} \psi(x, \omega) h(s) d s-\int_{t-T_{4}}^{t} M_{t-s}\left\{\frac{\sigma}{2} F^{\prime \prime}[\xi(s)] \tilde{u}(s)^{2}\right\}(x, \omega) d s \\
& +O\left(e^{-\rho t}\right) .
\end{aligned}
$$

$$
\begin{aligned}
M_{t-s}\left\{\frac{\sigma}{2} F^{\prime \prime}[\xi(s)] \tilde{u}(s)^{2}\right\}(x, \omega)= & \psi(x, \omega) h(s) \\
& +O\left(e^{-\rho(t-s)}\left\|\tilde{u}(s)^{2}\right\|\right),
\end{aligned}
$$

we have, | the second term of $(5.15) \mid \leq k_{6} \int_{0}^{t} e^{-\rho(t-s)} O\left(\left\|\tilde{u}(s)^{2}\right\|\right) d s$

$$
\leq k_{7} \int_{0}^{L}\left\|u(s)^{2}\right\| e^{-\rho(t-s)} d s+k_{8} \int_{L}^{t} \frac{e^{-\rho(t-s)}}{s^{2-2 \varepsilon}} d s=O\left(t^{2 \varepsilon-2}\right),
$$

where $L>1$ is a fixed sufficiently large number. Furthermore,

$$
\mid \text { the third term of }(5.15) \mid \leq k_{9} \int_{t-T_{4}}^{t} \frac{d s}{s^{2-2 \varepsilon}}=O\left(t^{2 \varepsilon-2}\right)
$$

and

$\mid$ the last term of $(5.15) \mid \leq k_{10} \int_{t-T_{4}}^{t} \frac{d s}{s^{2-2 \varepsilon}}=O\left(t^{2 \varepsilon-2}\right)$.

Hence,

$$
u(t, x, \omega)=\psi(x, \omega) \int_{t}^{\infty} h(s) d s+O\left(t^{2 \varepsilon-2}\right) .
$$

If we denote $G(t)=\int_{t}^{\infty} h(s) d s$, then $G(t)=O\left(t^{2 \varepsilon-1}\right)$ and

$$
u(t, x, \omega)=G(t) \psi(x, \omega)+O\left(t^{2 \varepsilon-2}\right) .
$$

Then,

$$
\begin{aligned}
G(t)= & \int_{t}^{\infty}\left\{\int_{G}\left(\sigma / 2 F^{\prime \prime}[1]+O\left(s^{\varepsilon-1}\right)\right)\right. \\
& \left.\times\left(G(s) \varphi(x)+O\left(s^{2 \varepsilon-2}\right)\right)^{2} \psi^{*}(x, \omega) d x d \omega\right\} d s \\
= & \int_{t}^{\infty} d s \int_{G}\left\{\frac{\sigma}{2} F^{\prime \prime}[1] G(s)^{2} \varphi(x)^{2}\left(1+O\left(s^{\varepsilon-1}\right)\right)\right. \\
& \left.+\frac{\sigma}{2} F^{\prime \prime}[1] G(s) \varphi(x) O\left(s^{2 \varepsilon-2}\right)+O\left(s^{3 \varepsilon-3}\right)\right\} \psi^{*}(x, \omega) d x d \omega .
\end{aligned}
$$

Let $a>0$ be as in Theorem 4.1. Then, there exists a positive constant $T_{5}$ such that, if $t \geqq T_{5}, e^{-\frac{t}{a}} \geqq\|g\|$. According to Theorem 4.1, there 
exist positive constants $k_{11}$ and $T_{6}$ such that, for every $t \geqq T_{6}$,

$$
u(t, x, \omega)=1-\left(T_{t} \hat{g}\right)(x, \omega) \geq 1-T_{t}\left(e^{-\frac{a}{t}}\right)(x, \omega) \geq \frac{k_{11}}{t} \psi(x, \omega) .
$$

Therefore,

$$
G^{-1}(s)=O(s), s \rightarrow \infty
$$

Since $\int_{G} \frac{\sigma}{2} F^{\prime \prime}[1] \varphi(x)^{2} \psi^{*}(x, \omega) d x d \omega$ is positive,

$$
G(t)=\int_{G} \frac{\sigma}{2} F^{\prime \prime}[1] \varphi(x)^{2} \psi^{*}(x, \omega) d x d \omega \cdot \int_{t}^{\infty} G(s)^{2} d s\left(1+O\left(t^{2 \varepsilon-1}\right)\right) .
$$

From Lemma 5.2, we have

$$
G(t)=\frac{1}{\int_{G} \frac{\sigma}{2} F^{\prime \prime}[1] \varphi(x)^{2} \psi^{*}(x, \omega) d x d \omega} \cdot \frac{1}{t}\left(1+O\left(t^{2 \varepsilon-1}\right)\right) .
$$

Consequently,

$$
u(t, x, \omega)=\frac{\phi(x, \omega)}{\int_{G} \frac{\sigma}{2} F^{\prime \prime}[1] \varphi(x)^{2} \psi^{*}(x, \omega) d x d \omega} \cdot \frac{1}{t}+O\left(t^{2 \varepsilon-2}\right)
$$

as desired.

§ 6. The Asymptotic Behaviour of $T_{t} \hat{g}(x, \omega)$ As $t \rightarrow \infty$, III:

Super-critical Case (i.e. $F^{\prime \prime}[1]>c^{*}$, or $\alpha>1$ )

In this case, the extinction probability $q(x, \omega)$ satisfies $0<q(x, \omega)<$ $1,(x, \omega) \notin \partial G$ and $q(x, \omega)=1,(x, \omega) \in \partial G$. We shall investigate the rate of convergence of $q(t, x, \omega)$ to $q(x, \omega)$, under the assumption that $F^{\prime \prime}[1]<\infty$.

Let $g \in \mathbf{C}_{1}^{+}$such that $g(x, \omega) \leq q(x, \omega)$. Then, by (1.3), $u(t, x, \omega)$ $\equiv q(x, \omega)-T_{t} \hat{g}(x, \omega)$ satisfies,

$$
\left\{\begin{array}{l}
\frac{\partial u(t, x, \omega)}{\partial t}=\omega \cdot \nabla u(t, x, \omega)-\sigma u(t, x, \omega)+\sigma\{F[\bar{q}(x)] \\
\quad-F[\bar{q}(x)-\tilde{u}(t, x)]\} \\
u(0+, x, \omega)=q(x, \omega)-g(x, \omega) \\
u(t, x, \omega)=0 \text { if }(x, \omega) \in \partial G .
\end{array}\right.
$$


In this case, we shall consider the following equation instead of (1.6),

$$
\left\{\begin{aligned}
\frac{\partial v(t, x, \omega)}{\partial t} & =\omega \cdot \nabla v(t, x, \omega)-\sigma v(t, x, \omega)+\sigma F^{\prime}[\tilde{q}(x)] \tilde{v}(t, x) \\
& \equiv \tilde{B} v(t, x, \omega) \\
v(0+, x, \omega)=f(x, \omega) & \\
v(t, x, \omega)=0 & \text { if }(x, \omega) \in \partial G .
\end{aligned}\right.
$$

Since there exists a positive constant $a$ such that $\tilde{q}(x) \geq a$, we can obtain as Lemma 2.3, the following estimate; if we denote the semigroup corresponding to (6.2) by $\tilde{M}_{t}$, then for every $f \in \mathbf{C}(G)$ (or $\mathfrak{S}(G)$ )

$$
\tilde{M}_{t} f(x, \omega)=e^{\gamma t}\left(f, \eta^{*}\right) \eta(x, \omega)+e^{\gamma t} O\left(e^{-\delta t}\|f\|\right),
$$

where $\delta$ is a positive constant, $\gamma$ is the eigenvalue of $B$ with maximal real part and $\eta\left(\eta^{*}\right)$ is the eigenfunction of $\tilde{B}$ (resp. $\tilde{B}^{*}$ ) corresponding to $\gamma$. The precise meaning of the above estimate is same as Lemma 2.3. Moreover, we can prove $\gamma<0$, in the following way.

Let $\tilde{E}(\lambda ; x, y)$ be the function $\frac{\sigma F^{\prime}[\tilde{q}(y)] e^{-(\sigma+\lambda) \mid x-y) \mid}}{4 \pi|x-y|^{2}}$, and $\tilde{E}(\lambda)$ be the integral operator on $\mathbf{C}(D)$ induced by this kernel. Then

$$
\left\{\begin{array}{l}
\tilde{B} f=\lambda f \\
f(x, \omega)=0,(x, \omega) \in \partial G
\end{array} \quad \text { if and only if } \tilde{E}(\lambda) \tilde{f}=\tilde{f} .\right.
$$

If we denote by $\mu_{1}(\beta)$ the largest eigenvalue of the operator $\tilde{E}(\beta)$, $\beta \in R^{1}$, then as in $[6],[9], \mu_{1}(\beta)$ is continuous, strictly decreasing, tends to zero as $\beta \rightarrow \infty$ and tends to infinity as $\beta \rightarrow-\infty$. Furthermore, 1 is the largest eigenvalue of $\tilde{E}(\lambda)$, i.e. $\tilde{\mu}_{1}(\lambda)=1$. Since $\tilde{q}(x)$ satisfies $\tilde{q}(x) \leq b$ by some constant $b<1$, there exists a positive constant $\varepsilon$ such that

$$
(1-\varepsilon)(1-\tilde{q}(x)) \geqq \tilde{E}(0)(1-\tilde{q})(x) .
$$

Then it follows from the following lemma that $\mu_{1}(0)<1$, and therefore $r<0$.

Lemma 6.1. (Karlin [5]) Suppose that $E$ is completely continuous and strictly positive operator on a Banach lattice, then the largest eigenvalue $r$ of $E$ is given by, 


$$
\gamma=\inf \left\{\lambda ;{ }^{\exists} x \neq 0, x \geq 0, E x \leq \lambda x\right\} .
$$

Now, we can prove the following theorem in the same way as the proof of Theorem 3.1.

Theorem 6.1. If we set $\inf _{(x, \omega)} q(x, \omega)=c>0$, then for every $g \in \mathbf{C}_{1}^{+}(G)$ such that $g \leq c$,

$$
g(x, \omega)-T_{t} \hat{g}(x, \omega)=\Gamma(g) e^{\gamma t} \tilde{\psi}(x, \omega)+e^{\gamma t} O\left(e^{-\delta t}\right)
$$

where $\delta$ is positive constant and $\Gamma(\mathrm{g})$ is positive constant depending on $g$.

\section{§ 7. Some Limit Theorems for Number of Particles}

First, we consider the supercritical case. Then, the extinction probability is non-trivial, that is, there is a positive probability that neutrons do not vanish and the number of them tends to infinity.

We shall discuss the asymptotic behaviour of the distribution of the neutrons under the assumption that $F^{\prime \prime}[1]<\infty$.

By the general theory of branching Markov processes $([2])$, we have,

$$
\begin{aligned}
& E_{(x, \omega)}\left(\check{f}\left(X_{t}\right) \check{g}\left(X_{t}\right)\right) \\
& =M_{t}(f g)(x, \omega)+\sigma F^{\prime \prime}[1] \int_{0}^{t} M_{t-u}\left\{\tilde{M}_{u} f \cdot \tilde{M}_{u} g\right\}(x, \omega) d u,
\end{aligned}
$$

where,

$$
\tilde{M}_{s} f(x)=\frac{1}{4 \pi} \int_{\Omega} M_{s} f(x, \omega) d \omega .
$$

Therefore,

$$
\begin{aligned}
E_{(x, \omega)\left[\check{f}\left(X_{t}\right)^{2}\right]} & =M_{t} f^{2}(x, \omega)+\sigma F^{\prime \prime}[1] \int_{0}^{t} M_{t-u}\left\{\tilde{M}_{u} f\right\}^{2}(x, \omega) d u \\
& =O\left(e^{\alpha t}\right)+\sigma F^{\prime \prime}[1] \int_{0}^{t} M_{t-u}\left\{\tilde{M}_{u} f\right\}^{2}(x, \omega) d u .
\end{aligned}
$$

On the other hand, for every $s \geqq 0$, it follows from the Markov and the branching property of the process $X_{t}$, that

$$
\begin{aligned}
E_{(x, \omega)}\left[\check{f}\left(X_{t}\right) \check{g}\left(X_{t+s}\right)\right] & =E_{(x, \omega)}\left[\check{f}\left(X_{t}\right) E_{X_{t}}\left(\check{g}\left(X_{s}\right)\right)\right] \\
& =E_{(x, \omega)}\left[\check{f}\left(X_{t}\right) \cdot M_{s} g\left(X_{t}\right)\right]
\end{aligned}
$$

By the definition of the $\tilde{M}_{t}$, 


$$
\tilde{M}_{u} \cdot M_{s}=\tilde{M}_{u+s} \cdot
$$

Hence, we have,

$$
\begin{aligned}
& E_{(x, \omega)}\left[\check{f}\left(X_{t}\right) \check{g}\left(X_{t+s}\right)\right]=M_{t}\left\{f \cdot M_{s} g\right\}(x, \omega) \\
& \quad+\sigma E^{\prime \prime}[1] \int_{0}^{t} M_{t-u}\left\{\tilde{M}_{u} f \cdot \tilde{M}_{u+s} g\right\}(x, \omega) d u .
\end{aligned}
$$

Now, let us define

$$
A(g) \equiv\left(g, \psi^{*}\right) /\left(1, \psi^{*}\right)
$$

and

$$
W_{t}(g)=\check{g}\left(X_{t}\right) / e^{\alpha t} A(g),
$$

where, $\psi^{*}$ is the eigenfunction of $B^{*}$ corresponding to $\alpha$. If $g$ is an indicator function of a set $E$, we write $A(g)$ as $A(E)$ and $W(g)$ as $W(E)$. Then

$$
\text { (7.8) } \begin{aligned}
& E_{(x, \omega)}\left[\left(W_{t}(f)-W_{t+s}(g)\right)^{2}\right]=\int_{0}^{t} M_{t+s-u}\left\{\tilde{M}_{u} g\right\}^{2}(x, \omega) d u / A(g)^{2} e^{2 \alpha(t+s)} \\
& +\int_{0}^{t} M_{t-u}\left\{\tilde{M}_{u} f / A(f) e^{\alpha t}-\tilde{M}_{u+s}(g) / A(g) e^{\alpha(t+s)}\right\}^{2}(x, \omega) d u \\
& +O\left(e^{-\alpha t}\right) \equiv R_{1}+R_{2}+O\left(e^{-\alpha t}\right) .
\end{aligned}
$$

For $R_{1}$,

$$
\begin{aligned}
& \int_{0}^{s} M_{t+s-u}\left\{\tilde{M}_{u} g\right\}^{2}(x, \omega) d u=\int_{0}^{T_{0}}+\int_{T_{0}}^{s} \\
& =O\left(e^{\alpha(t+s)}\right)+\int_{T_{0}}^{s} M_{t+s-u}\left\{\varphi(x)^{2} e^{2 \alpha u}\left(g, \phi^{*}\right)^{2}+e^{2 \alpha u} O\left(e^{-\rho u}\right)\right\}(x, \omega) d u \\
& \left.\leq O\left(e^{\alpha(t+s)}\right)+\int_{0}^{s} e^{\alpha(t+s-u}\right)\left\{e^{2 \alpha u}\left(g, \psi^{*}\right)^{2}\left(\varphi^{2}, \psi^{*}\right)\right. \\
& \left.\quad+e^{\alpha(t+s-u)+2 \alpha u} O\left(e^{-\rho(t+s)}\right)\right\} d u \\
& =O\left(e^{\alpha(t+2 s)}\right)
\end{aligned}
$$

where, $T_{0}$ and $\rho$ are the constants determined by Lemma 2.3 and we may assume that $2 \rho<\alpha$.

Therefore,

$$
R_{1}=\left(O e^{-\alpha t}\right) .
$$

For $R_{2}$, we set, 


$$
R_{2}=\int_{0}^{T^{0}}+\int_{T_{0}}^{t-T_{0}}+\int_{t-T_{0}}^{t}=R_{21}+R_{22}+R_{23}
$$

Since,

$$
\frac{\tilde{M}_{v} h}{A(h) e^{\alpha v}}=\frac{e^{\alpha v}\left(h, \psi^{*}\right) \varphi+e^{\alpha v} O\left(e^{-\rho v}\|h\|\right)}{\left(h, \phi^{*}\right) e^{\alpha v}}=\varphi+O\left(e^{-\rho v}\|h\|\right)
$$

there exists a constant $k_{1}$, such that, for every $h$ in some bounded set,

$$
\left|\frac{\tilde{M}_{v} h}{A(h) e^{\alpha v}}\right| \leq k_{1} \text {. }
$$

Then, it follows,

$$
R_{21} \leqq \frac{\left(1, \psi^{*}\right)^{2}}{e^{2 \alpha t}} \int_{0}^{T_{0}} e^{2 \alpha u} M_{t-u}\left\{4 k_{1}^{2}\right\}(x, \omega) d u=O\left(e^{-\alpha t}\right)
$$

Furthermore, we have

$$
\begin{aligned}
R_{22}= & \int_{T_{0}}^{t-T_{0}} M_{t-u}\left\{\frac{\left(f, \phi^{*}\right) e^{\alpha u} \cdot \varphi+O\left(e^{(\alpha-\rho) u}\right)}{A(f) \cdot e^{\alpha t}}\right. \\
& \left.--\frac{\left(g, \phi^{*}\right) e^{\alpha(u+s)} \varphi+O\left(e^{(\alpha-\rho)(u+s)}\right)}{A(g) \cdot e^{\alpha}}-\right\}^{2}(x, \omega) d u \\
\leqq & \left(1, \phi^{*}\right) \int_{T_{0}}^{t-T_{0}} M_{t-u}\left\{e^{2 \alpha(u-t)} O\left(e^{-\rho u}\right)\right\}(x, \omega) d u \\
\leqq & k_{2} \int_{0}^{t}\left\{e^{\alpha(u-t)} e^{-2 \rho u}\right\}(1+O(1)) d u=O\left(e^{-2 \rho u}\right)
\end{aligned}
$$

where, $k_{2}$ is some constant.

Similarly, we have

$$
\begin{aligned}
R_{23}= & \int_{t-T_{0}}^{t} M_{t-u}\left\{\frac{\left(f, \psi^{*}\right) e^{\alpha u} \varphi+e^{\alpha u} O\left(e^{-\rho t}\right)}{A(f) e^{\alpha t}}\right. \\
& \left.-\frac{\left(g, \phi^{*}\right) \varphi e^{\alpha(u+s)}+e^{\alpha(u+s)} O\left(e^{-\rho(u+s)}\right)}{A(g) e^{\alpha(t+s)}}\right\}^{2}(x, \omega) d u \\
\leqq & \left(1, \phi^{*}\right)^{2} e^{-2 \alpha t} \int_{t-T_{0}}^{t} k_{3} e^{2(\alpha-\rho) u} d u=O\left(e^{-2 \rho t}\right)
\end{aligned}
$$

where $k_{3}=\sup _{\substack{0 \leq u \leq T_{0} \\(x, \omega)}} M_{u}(1)(x, \omega)<+\infty$.

Consequently, we have proved the following lemma. 
Lemma 7.1. For every $f, g$

$$
E_{(x, \omega)}\left(W_{t}(f)-W_{t_{\top} s}(g)\right)^{2}=O\left(e^{-2 \rho t}\|f\| \vee\|g\|\right),
$$

uniformly in s.

Using this lemma, we can prove the following results as in Harris $[1]$, and $[10]$.

Theorem 7.1. There exists a non-trivial random variable $W$ such that $W_{t}(E)$ converges in the mean to $W$ for every $E$. And if we take a sequence $\left\{t_{n}\right\}$, so that $\sum e^{-2 \rho t_{n}}<+\infty$, then

$$
P_{(x, \omega)}\left(W_{t_{n}}(E) \rightarrow W\right)=1 .
$$

\section{Lemma 7.2.}

$$
\{W>0\}=\left\{e_{\partial}<+\infty\right\} \text { a.s. } P_{(x, \omega)}, \text { for every }(x, \omega) \in G .
$$

Theorem 7.2. For a sequence $\left\{t_{n}\right\}$ as in Theorem 7.1 and for $E \subset G$,

$$
P_{(x, \omega)}\left\{Z_{t_{n}}^{E} / Z_{t_{n}} \rightarrow A(E), \quad n \rightarrow \infty \mid e_{\partial}<+\infty\right\}=1 .
$$

for every $(x, \omega) \in G$.

Next, we consider the critical case. Then we can derive the following theorem from Theorems 4.1 and 5.1.

Theorem 7.3. For every $E_{1}, E_{2}, \ldots E_{n} \subset G$, such that $\left(I_{E_{\imath}}, 1\right)>0$ $(i=1,2, \ldots, n)$, the joint distribution of $\left[\frac{t}{2} \sigma F^{\prime \prime}[1]\left(\tilde{\psi}^{2}, \psi^{*}\right)\right]^{-1}\left(Z_{t}^{E_{1}}, Z_{t}^{E_{2}}, \cdots\right.$, $\left.Z_{t}^{E_{n}}\right)$, under the condition $Z_{t}^{G} \neq 0$, converges to that of $\left(\left(I_{E_{1}}, \phi^{*}\right),\left(I_{E_{2}}, \phi^{*}\right)\right.$, $\left.\ldots\left(I_{E_{n}}, \psi^{*}\right)\right) \cdot W$ when $t \rightarrow \infty$, where $W$ is exponentially distributed with mean 1.

\section{References}

[1] Harris, T.E., The Theory of Branching Processes, Springer, 1963.

[2] Ikeda, N., M. Nagasawa and S. Watanabe., Branching Markov processes I. II. 
III., J. Math. Kyoto Univ. 8 (1968), 233-278, 364-410; ibid. 9 (1969), 95-160.

[3] Jorgens, K., An asymptotic expansion in the theory of neutron transport, Comm. Pure Appl. Math. 11 (1958) 219-242.

[4] Karlin, S., Positive operators, J. Math. Mech. 8 (1959) 907-937.

[5] Krein, M.G. and M.A. Rutman, Linear operators leaving invariant a cone in Banach space, Amer. Math. Soc. Transl. 26 (1950).

[6] Mori, T., Neutron transport process on bounded homogeneous domain, Proc. Japan Acad. 46 (1970), 944-948.

[7] Mullikin, T.W., Branching processes in neutron transport theory, Probabilistic Method in Applied Mathematics, Vol. I edited by Barucha-Reid, Academic Press, 1968.

[8] Pazy A. and P.H. Rabinowitz, A nonlinear integral equation with applications to neutron transport theory, Arch. Rat. Mech. Anal. 32 (1969) 226-247.

[9] Ukai, S., Real eigenvalues of the monoenergetic transport operator for a homogeneous medium, J. Nucl. Sci. Technol. 3 (1966), 263-266.

[10] Watanabe, S., On the branching process for Brownian particles with an absorbing boundary. J. Math. Kyoto Univ. 4 (1965) 385-398. 
